\title{
A Consideration on the Tidal Wave Transmission through the Ionized Atmosphere
}

\author{
Susumu KAto* and Sadami Matsushita \\ High Altitude Observatory, National Center for Atmospheric Research, \\ Boulder, Colorado 80302.
}

(Received May 6, 1969)

\begin{abstract}
We investigate the effect of $\left(\boldsymbol{J} \times \boldsymbol{B}_{0}\right)$ force on the tidal wave transmission where $\boldsymbol{J}$ is electric current and $\boldsymbol{B}_{0}$ the geomagnetic field. It is found that the force is considerably effective on any positive mode of the diurnal tide and the semi-diurnal tide except for the first semi-diurnal mode. The diurnal and semi-diurnal velocity oscillations of these modes, which are excited in the lower neutral atmosphere, cannot be transferred to the ionized atmosphere. An exception occurs, however, at the magnetic equator, where $\left(\boldsymbol{J} \times \boldsymbol{B}_{0}\right)$ force is almost ineffective. The refractive index of the tidal wave is calculated for a simple model where the Coriolis force is ignored and $\left(\boldsymbol{J} \times \boldsymbol{B}_{0}\right)$ is assumed to be uniform with longitude and latitude.
\end{abstract}

\section{Introduction}

The upper atmosphere, approximately $90 \mathrm{~km}$ above the ground, is ionized and electrically conductive. The air motion in this region interacts with the geomagnetic field, thereby producing an electromotive force. The electric current driven by this electromotive force produces a magnetic field variation which is detected on the ground as geomagnetic variation. The electric current further produces a mechanical force, the Ampère force, which may modify the original air motion. The time constants associated with this force become $24 \mathrm{hrs}$ or $12 \mathrm{hrs}$ in the E region (Fig. 1) and less in higher region. It is expected, then, that an air motion with a time scale equal to or larger than these periods should be affected by this force in these regions. The air motion associated with the diurnal and semi-diurnal tides belongs to such category.

In this paper the main concern is as to how the thermotidal energy, which is originally supplied in the lower neutral atmosphere, is transferred to and above the lower ionosphere. The theory developed here must be regarded as a preliminary step in that the treatment is linear and the Coriolis force and the viscosity are neglected. Nevertheless, it may reveal some basic effects of the ionosphere upon the tidal wave transmission.

It should be remarked that the introduction of the Coriolis force gives negative as well as positive modes for the atmospheric tide (Kato, $1966 \mathrm{a}, \mathrm{b}$, and Lindzen, 1966). The present concern is on the positive mode only which can transfer the energy vertically. The equivalent depth for this mode in the Laplace tidal equation is increased in the absence of the Coriolis force, as will be shown in the following section.

* Present Address : Ionosphere Research Laboratory, Kyoto University, Kyoto, JAPAN. 


\section{Basic Relations}

The notations used are as follows:

$a=$ the earth's radius

$g=$ acceleration of gravity

$\Omega=$ the earth's angular velocity

$u, v$, and $w=$ southward, eastward and upward air velocity respectively; $\boldsymbol{U}=(u, v, w)$

$t=$ time

$p=$ pressure $;$ static part $p_{0}$ plus perturbation $p_{1}$, $p=p_{0}+p_{1}$

$\rho=$ density $;$ as in $p, \rho=\rho_{0}+\rho_{1}$

$\theta=$ co-latitude

$\phi=$ longitude

$z=$ (vertically upward) height

$\boldsymbol{J}=\left(J_{\theta}, J_{\phi}, J_{z}\right)=$ electric current density in e.m.u.

$\boldsymbol{E}=\left(E_{\theta}, E_{\phi}, E_{z}\right)=$ electric field

$\boldsymbol{B}_{0}=\left(B_{\theta}, 0, B_{z}\right)=$ geomagnetic field of a dipole placed at the earth's center and along the rotation axis

$\sigma_{0}, \sigma_{1}$, and $\sigma_{2}=$ electrical conductivity, parallel, Pedersen, and Hall conductivities respectively

$T=$ temperature; as in $p$ and $\rho, T=T_{0}+T_{1}$

$R=$ specific gas constant

$H=$ scale height $=R T_{0} / g$

$C=$ sound velocity $=\left(\gamma p_{0} / \rho_{0}\right)^{1 / 2}=\left(\gamma R T_{0}\right)^{1 / 2}$

$\omega=$ angular frequency of oscillation

$\gamma=$ ratio of heat capacity

The dynamical equations of motion for weak perturbations in otherwise static ionized atmosphere are

$$
\begin{aligned}
\rho_{0} \frac{\partial u}{\partial t} & =J_{\phi} B_{z}-\frac{1}{a} \frac{\partial p_{1}}{\partial \theta} \\
\rho_{0} \frac{\partial v}{\partial t} & =-J_{\theta} B_{z}-\frac{1}{a \sin \theta} \frac{\partial p_{1}}{\partial \phi} \\
\frac{d p_{0}}{d z} & =-g \rho_{0} \\
\frac{\partial p_{1}}{\partial z} & =-g \rho_{1}
\end{aligned}
$$

where $J_{z}$ is neglected (e.g. Maeda and Murata, 1965); the vertical acceleration and Ampère force are also neglected. The continuity equation is

$$
\frac{\partial \rho_{1}}{\partial t}+w \frac{\partial \rho_{0}}{\partial z}+\rho_{0} \boldsymbol{\nabla} \cdot \boldsymbol{U}=0
$$

where production and loss of plasma are neglected. The adiabatic condition is 


$$
\frac{\partial p}{\partial t}+(\boldsymbol{U} \cdot \boldsymbol{\nabla}) p_{0}=C^{2}\left[\frac{\partial \rho_{1}}{\partial t}+\boldsymbol{U} \cdot \boldsymbol{\nabla} \rho_{0}\right]
$$

where joule heating is neglected because it is of second order in magnitude.

In addition to (2.1)-(2.3), the electromagnetic relations are as follows:

$$
\boldsymbol{\nabla} \times \boldsymbol{E}=0
$$

which is valid, because the phase velocity of tide is much smaller than that of the Alfvén wave. The displacement current is negligible and we have

$$
\boldsymbol{\nabla} \cdot \boldsymbol{J}=0
$$

$\boldsymbol{J}$ and $\boldsymbol{E}$ are related by Ohm's law as

$$
\boldsymbol{J}=[\sigma]\left(\boldsymbol{E}+\boldsymbol{U} \times \boldsymbol{B}_{0}\right)
$$

where $[\sigma]$ is conductivity tensor of $\sigma_{0}, \sigma_{1}$, and $\sigma_{2}$ (e.g. Maeda and Kato, 1966).

Since $\boldsymbol{E}$ is electrostatic as shown by (2.4), $\boldsymbol{E}$ is caused only by accumulation of electric charge which is transported by the dynamo electromotive force $\left(\boldsymbol{U} \times \boldsymbol{B}_{0}\right)$. However, high conductivity along $\boldsymbol{B}_{0}, \sigma_{0}$, may produce a rapid discharge along the geomagnetic field line, because $\left(\boldsymbol{U} \times \boldsymbol{B}_{0}\right)$ changes sign with distance along the field lines. Then, $|\boldsymbol{E}| \ll\left|\boldsymbol{U} \times \boldsymbol{B}_{0}\right|$ is expected (Appendix) except at the equator where $\boldsymbol{E}$ can be large because the tidal wave travels perpendicularly to $\boldsymbol{B}_{0}$, and no change in phase of $\left(\boldsymbol{U} \times \boldsymbol{B}_{0}\right)$ occurs along $\boldsymbol{B}_{0}$. A further simplification is to assume that $\left(\boldsymbol{J} \times \boldsymbol{B}_{0}\right)$-force is vertically and horizontally uniform. This assumption is fairly unrealistic, but necessary to find a simple solution of the probelm which is attempted here. Variations of $\left(\boldsymbol{J} \times \boldsymbol{B}_{0}\right)$ with $\theta$, $\phi$, or $z$ may induce a complicating coupling among various modes of the tide, which is avoided in this paper.

In (2.1) $\left(\rho_{0}^{-1} J_{\phi} B_{z}\right)$ and $\left(\rho_{0}^{-1} J_{\theta} B_{z}\right)$ are replaced by $(-\alpha u+\beta v)$ and $(-\beta u-\alpha v)$ respectively where

$$
\begin{aligned}
& \alpha=\frac{\sigma_{1} B_{0}^{2}}{\rho_{0}} \\
& \beta=\frac{\sigma_{2} B_{0}^{2}}{\rho_{0}}
\end{aligned}
$$

This approximation is poor except at $\theta=0$ where the replacement is strict. Fig. 1 illustrates $\alpha$ and $\beta$ for a model atmosphere with $\sigma_{1}$ and $\sigma_{2}$ at $12 \mathrm{hrs}$ (Maeda and Matsumoto, 1962) and $B_{0}=0.33$ e.m.u. It is found that $\alpha$ and $\beta$, which have dimensions of frequencies, amount to the frequencies equal to those of diurnal or semi-diurnal oscillation between $120 \mathrm{~km}$ and $140 \mathrm{~km}$ heights. Above these heights, $\alpha$ increases with height and tends to be about $1 \mathrm{hr}^{-1}$, while $\beta$ once reaches a maximum as a few $\mathrm{hrs}^{-1}$ around $140 \mathrm{~km}$ height and then decreases with height. The first two equations in (2.1) shows that if $\alpha$ and $\beta$ are equal to or larger than $\omega$, the $\left(\boldsymbol{J} \times \boldsymbol{B}_{0}\right)$ force affects the motion of the atmosphere appreciably. Such a frequency of $\omega$ is called a low frequency. We may call $\alpha$ and $\beta$ the charateristic frequency of the ionized gas. If $\omega$ is much larger than $\alpha$ and $\beta$, the $\left(\boldsymbol{J} \times \boldsymbol{B}_{0}\right)$ force is unimportant in the motion of the atmosphere as a whole i.e. mainly the neutral gas in the ionosphere. Such $\omega$ is called a high-frequency and found in fluctuation of electron density as of many TID's (travelling 


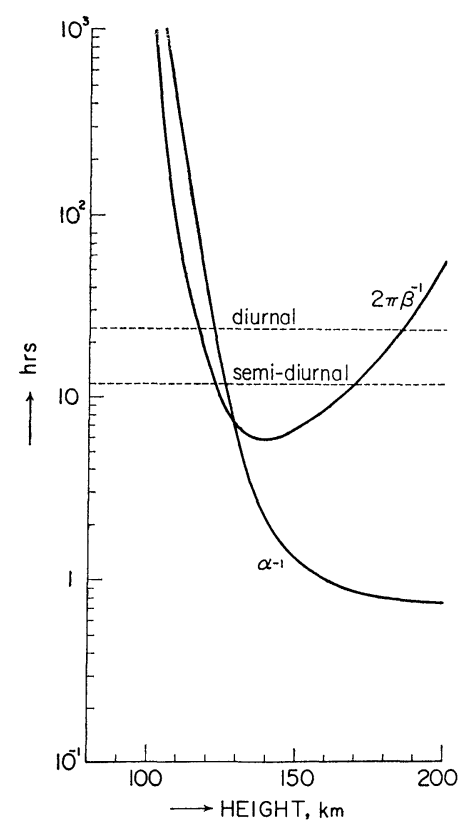

Fig. 1 Characteristic frequency: $\alpha$ and $\beta$

The dotted lines dencte the diurnal and semi-diurnal periods. For $\alpha$, the curve shows $\alpha^{-1}$, and for $\beta$ the curve shows $2 \pi \beta^{-1}$ because $\alpha^{-1}$ gives $e^{-1}$-fold decay time, and $2 \pi \beta^{-1}$ is period (Piddington, 1954). ionosphere disturbances) and radio-wave scintillation or fading. In these high-frequency phenomena, the movement of ionization is strongly affected by the neutral-gas movement, but the reaction of ionization upon neutral gas is practically ignored. Note, however, that the peculiar motion of ionization for such a high frequency is important in explaining various ionospherc behaviour (e.g. Maeda and Kato, 1966). The boundary between the high and the low frequency varies with height and time, but the maximum of $\alpha$ and $\beta$ corresponds approximately to $1 \mathrm{hr}$ (Fig. 1) showing that a perturbation with longer period than $1 \mathrm{hr}$ cannot be freely transmitted through the ionosphere; a strong reflection and absorption can be expected.

Since we are concerned with diurnal or semi-diurnal variation, time and longitude variations are assumed to be proportional to $\exp [j s(\Omega t+\phi)]$ where $s=1,2$ corresponding to the diurnal and semi-diurnal oscillation. From (2.1)

$$
\begin{aligned}
& u=\left(\frac{j}{a}\right) \frac{(s \Omega-j \alpha)}{(s \Omega-j \alpha)^{2}-\beta^{2}}\left[\frac{\partial}{\partial \theta}+\frac{\beta}{s \Omega-j \alpha} \frac{s^{2}}{\sin \theta}\right]\left(\frac{p_{1}}{\rho_{0}}\right) \\
& v=-\left(\frac{1}{a}\right) \frac{(s \Omega-j \alpha)}{(s \Omega-j \alpha)^{2}-\beta^{2}}\left[\frac{\beta}{s \Omega-j \alpha} \frac{\partial}{\partial \theta}+\frac{s}{\sin \theta}\right]\left(\frac{p_{1}}{\rho_{0}}\right)
\end{aligned}
$$

Following, then, usual mathematical approach as in the theory of atmospheric oscillation (e.g. Wilkes, 1949), we finally get from (2.2), (2.3), and (2.7)

$$
\begin{gathered}
L(\Theta)+\frac{a^{2} \Omega^{2} s^{2}}{g h} \Theta=0 \\
C^{2} \frac{d^{2} Y}{d z^{2}}+\left[\frac{d c^{2}}{d z}-\gamma g\right] \frac{d Y}{d z}+\frac{Y A}{h}\left[\frac{d C^{2}}{d z}+g(\gamma-1)\right]=0
\end{gathered}
$$

where $h$ is a separation constant; $\Theta$ and $Y$ are functions of only $\theta$ and $z$, respectively; $\{\Theta Y \exp [j s(\Omega t+\phi)]\}$ gives $\boldsymbol{\nabla} \cdot \boldsymbol{U}$ in terms of which we can express any perturbation as $\left(p_{1} / \rho_{0}\right)$ being proportional to $\boldsymbol{\nabla} \cdot \boldsymbol{U}$.

$$
\begin{aligned}
& L \equiv \text { Legendre's operator }=\frac{1}{\sin \theta} \frac{d}{d \theta}\left(\sin \theta \frac{d}{d \theta}\right)-\frac{s^{2}}{\sin ^{2} \theta} \\
& A=\frac{1-j \frac{\alpha}{s \Omega}}{\left(1-\frac{j \alpha}{s \Omega}\right)^{2}-\frac{\beta^{2}}{s \Omega}}
\end{aligned}
$$

The first equation in (2.9) is the same to that in the absence of conductivity on a nonrotating planet; the second is also the same to the Wilkes equation except for $A$, which depends on conductivities. 
The first equation in (2.9) shows that $\Theta$ is a linear combination of eigen functions, each of which is now an associated Legendre function $P_{n}^{s}(\theta)$ with order $n$ and degree $s$ and the eigen values $h_{n}^{s}$ or $\alpha_{n}^{s}$ are given by

$$
\alpha_{n}{ }^{s}=\frac{g h_{n}{ }^{s}}{4 a^{2} \Omega^{2}}=\frac{s^{2}}{4 n(n+1)}
$$

where $n$ is (positive) integer, which is necessary for $\Theta$ to be finite at $\theta=180^{\circ}$. Note that consideration of the Coriolis force replaces the equation by the Laplace tidal equation which posseses both positive and negative $h$ 's (Kato, $1966 \mathrm{a}, \mathrm{b}$ ). While the negative mode has no counterpart in the absence of the Coriolis force, the positive mode exists also in this case. For the diurnal tide, the first positive mode is fairly similar to $P_{3}{ }^{1}$ or $P_{5}{ }^{1}$ (Kato, $1966 \mathrm{a}, \mathrm{b})$ in shape having $g h_{1}{ }^{1} / 4 a^{2} \Omega^{2}=1 / 125$ from the exact Lapalce tidal equation, and (2.12) gives $\alpha_{3}{ }^{1}=1 / 48$ and $\alpha_{5}{ }^{1}=1 / 120$; similarly for the semi diurnal tide, the symmetric first positive mode has, as the exact value, $g h_{2}{ }^{2} / 4 a^{2} \Omega^{2}=1 / 11$, and (2.12) gives $\alpha_{2}{ }^{2}=1 / 6$ and $\alpha_{4}{ }^{2}=1 / 20$. Tranformation $d z=H d x$ and $Y=y e^{x / 2}$ gives

$$
\frac{d^{2} y}{d x^{2}}+\left[-\frac{1}{4}+\left(\frac{H}{h}\right) A\left(\frac{1}{H} \frac{d H}{d x}+\frac{r-1}{r}\right)\right] y=0
$$

which describes propagation of the tidal wave away the height of excitation; this is of the present concern. In (2.11) $A$ tends to unity with decreasing $\alpha$ and $\beta$; $(2.13)$ on this limit reduces to the Wilkes equation.

\section{Reflection and Absorption of Tidal Wave}

By analogy to electromagnetic wave, we introduce the refractive index $N$ which is defined by (2.13) as follows;

$$
\frac{d^{2} y}{d x^{2}}+N^{2} y=0
$$

where

$$
N=\sqrt{-\frac{1}{4}+A \frac{H}{h}\left(\frac{1}{H} \frac{d H}{d x}+\frac{r-1}{r}\right)}
$$

Since $(\alpha / s \Omega)^{2}$ is much larger than unity above $150 \mathrm{~km}$, the following asymptotic expression of $A$ is obtained by (2.11):

$$
A=\frac{1}{r^{2}}+j \frac{1}{r}
$$

where $r=\alpha / s \Omega$. Fig. 2 illustrates how $N$ varies with $R$ for $r=5$ where $R=(H / h)[(1 / H)(d H / d x)+(\gamma-1) / \gamma]$. The altitude with such a large $r$ is about $150 \mathrm{~km}$ and the diagram shows both real part, $R_{e}$, and imaginary part, $I_{m}$, of $N$ and, for comparison, $N$ in the absence of conductivity by the dotted curve. Consider a wave with $h_{n}{ }^{s}$ travelling upwards after excitation. If $I_{m}(N)$ for the wave exceeds $0.5, Y$, equal to $y e^{x / 2}$, tends to decrease with height, so do $u$ and $v$, because $u$ and $v$ are proportional to $Y$ (e.g. Wilkes, 1949). The de- 


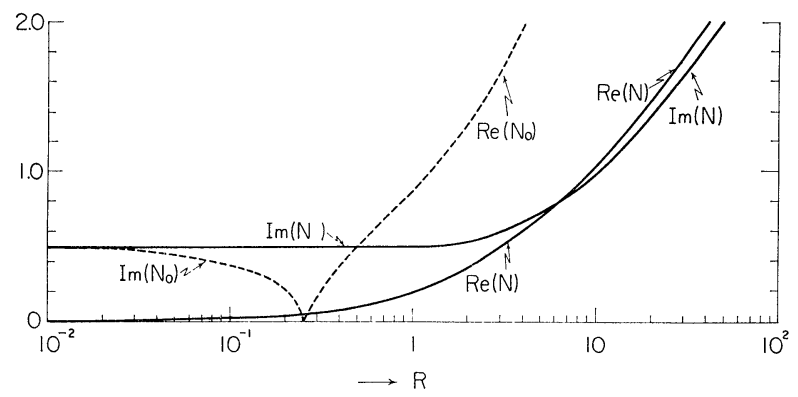

Fig. 2 Refractive index, $N$, as a function of $\mathrm{R} . R_{e}(N)$ and $I_{m}(N)$ denote real and imaginary part. For comparison, $N$ in the absence of electrical conductivity is given by the dotted curve.
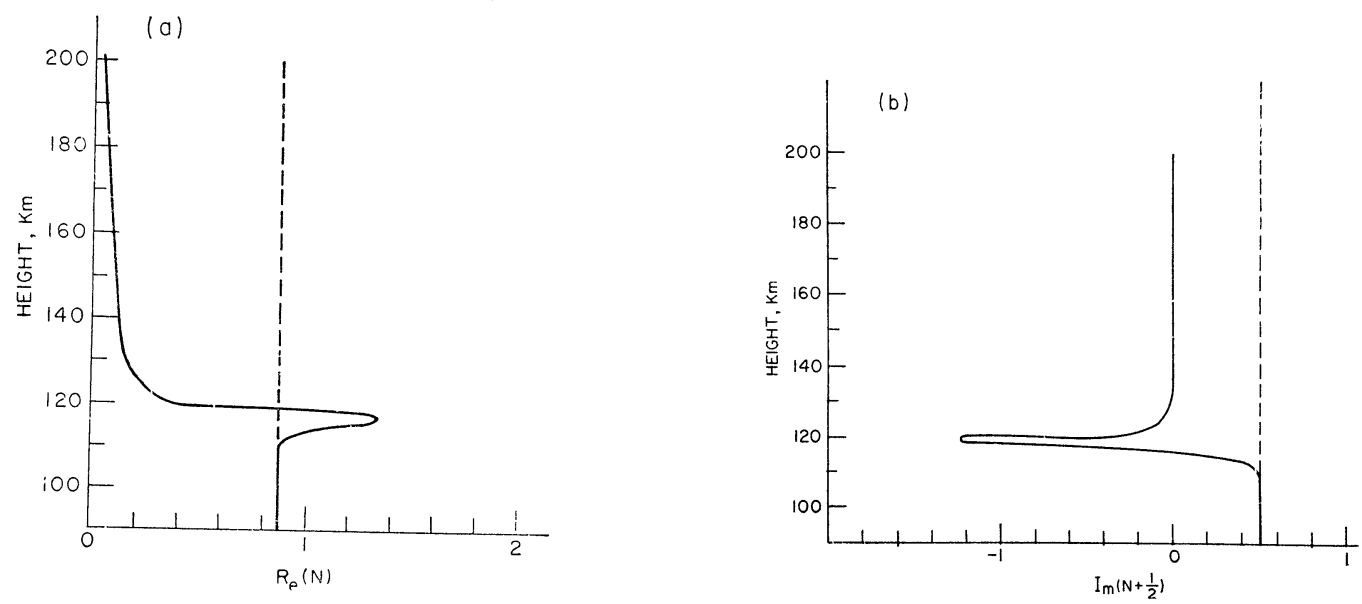

Fig. 3 Refractive index, $N$, as a function of height and for $R=1$, which gives the threshold of attenuation. (a) gives $R_{e}(N)$ and (b) $I_{m}(N)$.

creasing density $\rho_{0}$ with height is overcome by decreasing $p_{1}$ resulting in decreasing $\left(p_{1} / \rho_{0}\right)$. The kinetic energy density per unit mass, $\boldsymbol{U}^{2}$, decreases with height. The threshold for such decrease is $R=1$. Since $R$ is inversely proportional to $h_{n}^{s}$ in (3.2), the smaller $h_{n}{ }^{s}$ produces the stronger attenuation.

For $H=8 \mathrm{~km},(1 / H)(d H / d x)=0.2$ and $\gamma=1.4$, the threshold of $h$ for $R=1$ is $h_{0}$ as

$$
h_{0} \approx \frac{H}{2}=4 \mathrm{~km}
$$

Any mode for the diurnal tide has $h_{n}{ }^{1} \ll h_{0}$ as given by (2.12) or by a rigorous calculation (e.g. Kato, 1966a, b). It is concluded that the diurnal tide attenuates strongly with height. As to the semi-diurnal tide, the first mode has $h_{2}{ }^{2}$ of about $8 \mathrm{~km}$, much larger than $h_{0}$, but other modes have $h$ smaller than $h_{0}$; the semi-diurnal tide of only the first mode travels freely through the ionosphere.

In the lower ionosphere $r^{2}$ can be smaller than or equal to unity, and (2.11) must be used instead of its asymptotic formula (3.3) for large $r^{2}$. We shall consider a simple case assuming an isothermal atmosphere and find $N$ for the wave with $h_{0}$. Fig. 3 illustrates 
both real and imaginary parts of such $N, R_{e}(N)$ and $I_{m}(N)$ respectively for $s=1$; a similar result will be obtained for $s=2$. Note that a maximum appears in $R_{e}(N)$ and $I_{m}(N)$ at a height at which $\beta=\Omega$ is approximately valid.

\section{Discussion}

The excitation of wind motion in the thermosphere has recently been discussed by Volland (1966) and Dickinson et al. (1968). They are mainly concerned with heating by the solar radiation absorbed in the thermosphere. It is, however, no less important to investigate the supply of the dynamical energy flux flowing into the thermosphere from the lower region. The present work shows that the electrical conductivity tends to attenuate the tidal motion, which is excited in the lower neutral atmosphere and propagates upwards to the thermosphere. The attenuation is so large that any mode of the diurnal tide and of the semi-diurnal tide except for the semi-diurnal first mode $h_{2}{ }^{2} \approx 8 \mathrm{~km}$ can hardly reach the F region. It is very likely that the diurnal wind oscillation as deduced by Lindzen (1967) and Geisler (1967) from observation is excited in the thermosphere by solar radiation. Another possibility is the excitation taking place far above the F-region and transferred downwards to the region in the form of hydromagnetic waves (Poeverlein, 1966).

The attenuation occurs mainly by ion drag, which is considered in the form of $\alpha$ in (2.7). Fig. 3 shows a sharp minimum of $I_{m}(N)$ approximately at $120 \mathrm{~km}$ at which $\beta$ is as large as $s \Omega$. The dissipation by such $\beta$ is interpreted by absorption of the plasma which tends to rotate around the lines of force of $\boldsymbol{B}_{0}$ with the characteristic frequency $\beta$ (Piddington, 1954) and resonates with the incident wave with frequency $s \Omega=\beta$. It is interesting to note that just below the height with this minimum of $I_{m}(N)$ we have a sharp maximum of $R_{e}(N)$, which suggests the existence of reflection. However, these maximum and minimum are so narrow in width that their effect on the wave transmission is not appreciable.

The present work is based on various simplification, some of which are fairly unrealistic. Neglect of electric field becomes considerably erroneous in the vicinity of the magnetic equator where the tidal wave travels almost orthogonally to the magnetic field producing the polarization field orthogonal to the magnetic field. This electric field is set up in such a way that the electromotive force $\left(\boldsymbol{U} \times \boldsymbol{B}_{0}\right)$ tends to be balanced, resulting in decreasing $\left(\boldsymbol{J} \times \boldsymbol{B}_{0}\right)$ force. If the wave front of tide is exactly parallel to $\boldsymbol{B}_{0}$, the balance is complete and, hence, $\boldsymbol{J}=0$, which means transmission of the tidal wave free from electromagnetic fields at the equator. Except at the equator, the present calculation may give a slight overestimate of the effect by $\left(\boldsymbol{J} \times \boldsymbol{B}_{0}\right)$.

\section{Acknowledgement}

Kato read this paper at a seminar, Space Flight Center, NASA, Greenbelt, Maryland, while he was visiting Dr. Kaichi Maeda. We acknowledge interesting discussions with him and Dr. Volland at the seminar. 


\section{Appendix}

\section{Electrostatic field produced by $\left(\boldsymbol{U} \times \boldsymbol{B}_{0}\right)$}

Consider an electrostatic field produced by $\boldsymbol{E}_{0}=\boldsymbol{U} \times \boldsymbol{B}_{0}$ which is orthogonal to $\boldsymbol{B}_{0}$ and is assumed to vary harmonically with distance $\boldsymbol{r}$ as $\exp (j \boldsymbol{k} \cdot \boldsymbol{r})$ where $\boldsymbol{k}$ is the wave vector; $\boldsymbol{B}_{0}$ is along the $z$ axis and $\boldsymbol{E}_{0}$ is along the $y$ axis. Eq. (2.5) gives

$$
\varphi=\frac{j \sigma_{1} k_{y} E_{0}}{\sigma_{1} k_{y}^{2}+\sigma_{0} k_{z}^{2}}
$$

where $\varphi$ is the potential and $\boldsymbol{E}=\nabla \varphi=j \boldsymbol{k} \varphi$. And

$$
E_{y}=-\frac{\sigma_{1} k_{y}^{2}}{\sigma_{1} k_{y}^{2}+\sigma_{0} k_{z}^{2}} E_{0}
$$

Hence,

$$
\left|E_{y} / E_{0}\right|=\frac{\sigma_{1} k_{y}{ }^{2}}{\sigma_{1} k_{y}{ }^{2}+\sigma_{0} k_{z}{ }^{2}} \ll 1
$$

if $k_{z} / k_{y} \gg\left(\sigma_{1} / \sigma_{0}\right)^{1 / 2}$, which is true if $\boldsymbol{k}$ deviates from the normal to $\boldsymbol{B}_{0}$ only by a few degrees or more.

The model used here is very simple, but still can show in general how small $\boldsymbol{E}$ is produced by $\left(\boldsymbol{U} \times \boldsymbol{B}_{0}\right)$ at any latitude except at the magnetic equator.

\section{References}

Dickinson, R.E., Lagos, C.P., and Newell, R.E., 1968, Dynamics of the neutral gas in the thermosphere for small Rossby number motions, J. Geophys. Res. 73, 4299-4314.

Geisler, J.E., 1967, A numerical study of the wind system in the middle thermosphere, J. Atmosph. Terr. Phys. 29, 1469-1482.

Kato, S., 1966a, Diurnal atmospheric oscillation 1. Eigenvalues and Hough functions, J. Geophys. Res. 71, 3201-3210.

Kato, S., 1966b, Diurnal and semi-diurnal atmospheric tidal oscillation, Eigenvalues and Hough functions, Rep. Ionosph. Space Res. Japan 20, 448-463.

Lindzen, R.S., 1966, On the theory of the diurnal tide, Monthly Weather Rev. 94, 295-301.

Lindzen, R.S., 1967, Reconsideration of diurnal velocity oscillation in the thermosphere, J. Geophys. Res. 72, 1591-1598.

Maeda, K. and Kato, S., 1966, Electrodynamics of the ionosphere, Space Sci. Rev. 5, 57-79.

Maeda, K. and Matsumoto, H., 1962, Conductivity of the ionosphere and current system, Rep. Ionosph. Space Res. Japan 16, 1-26.

Maeda, K. and Murata, H., 1965, Ionospheric dynamo theory with consideration for magnetospheric current along the geomagnetic lines of force, Rep. Ionosph. Space Res. Japan 19, 272-285.

Piddington, J.H., 1954, The motion of ionized gas in combined magnetic, electric and mechanical fields of force, Mon. Not. Roy. Astronom. Soc. 114, 651-663.

Poeverlein, H., 1966, Geomagnetic and ionospheric effects of magnetospheric motions, J. Atmosph. Terr. Phys. 28. 1111-1123.

Volland, H., 1966, A two-dimensional dynamic model of the diurnal variation of the thermosphere, Pt. 1. Theory, J. Atmosph. Sci. 23, 799-807.

Wilkes, M.V., 1949, Oscillation of the earth's atmosphere, Cambridge Univ. Press. 